\title{
Les ennemis de la concurrence et de l'emploi
}

\author{
Pierre Cahuc \\ Francis Kramarz \\ André Zylberberg
}

Aux élections présidentielles de 2000, Georges Bush a conquis l'Etat de Virginie Occidentale, où étaient localisées de grandes entreprises sidérurgiques dont les salariés votaient traditionnellement pour le Parti démocrate, en promettant de limiter les importations d'acier. Une fois élu, Georges Bush tiendra sa promesse, alors que son prédécesseur démocrate, Bill Clinton, s'était toujours refusé à prendre ce genre de mesures. En fait, la mauvaise santé des grands conglomérats sidérurgiques nord-américains était le fruit d'une évolution prévisible. Entre 1980 et 2004, la productivité moyenne de la sidérurgie aux Etats-Unis avait pratiquement triplé entre 1980 et 2004, mais les grands conglomérats producteurs d'acier comme U.S. Steel étaient restés largement à la traîne de ce mouvement. Au début des années 2000, ils n'étaient plus capables de faire face à la concurrence des importateurs étrangers, mais aussi d'entreprises américaines plus petites (les mini-mills), nettement plus efficaces et parfaitement à même de résister à la concurrence internationale. Dans ce contexte, Georges Bush s'aligna sur les doléances des dirigeants des grands conglomérats et d'une partie de leurs 150000 salariés, qui menèrent quelques actions fortement médiatisées, en décidant de limiter les importations d'acier².

Faut-il féliciter Georges Bush d'avoir su éviter de nombreuses destructions d'emplois? Les évaluations menées a posteriori apportent une réponse clairement négative. Les barrières tarifaires ont détruit beaucoup plus d'emplois parmi les entreprises utilisatrices d'acier qu'elles n'ont permis d'en sauver parmi les producteurs de cette ressource. Ainsi, Gary Hufbauer et Ben Goodrich ont calculé que les barrières tarifaires ont préservé environ 3500 emplois chez les producteurs, mais en ont détruit entre 12000 et 43000 chez les entreprises utilisatrices ${ }^{3}$. Ces conséquences étaient prévisibles, et c'est pour cette raison que Bill Clinton avait refusé de céder à la pression des salariés et des dirigeants des grandes usines traditionnelles. Cette pression était d'autant plus forte que ces derniers avaient individuellement beaucoup à perdre de la nouvelle

\footnotetext{
1 Voir R. Rajan et L. Zingales, Saving capitalism from the capitalists, Princeton University Press, 2004, pp. 228-232.

2 Ce mécanisme est très souvent observé: la capacité de mobilisation politique des victimes est généralement plus grande que celle des bénéfidiaires. En outre, tous les ouvriers n'auraient pas été touchés mais tous étaient menacés.

3 Gary Hufbauer et Ben Goodrich, Steel Protection and Job Dislocation, Washington: Institute for International Economics. Février 2003.
} 
donne. En revanche, les neuf millions de travailleurs dont l'emploi actuel dépend peu ou prou de la fourniture d'acier avaient individuellement peu à y gagner. D e plus, les travailleurs qui auraient pu être embauchés par l'accroissement du marché permis par la baisse des prix de l'acier auraient pu gagner beaucoup, mais leurs voix restèrent et restent encore pour le moins virtuelles. Parmi les gagnants, il y aurait eu aussi tous les consommateurs qui achètent des produits où l'acier intervient dans la chaine de fabrication. Mais pour ceux-là le gain individuel est faible et la plupart n'en n'ont pas véritablement conscience.

Le comportement de Georges Bush est emblématique d'une tendance des autorités publiques à céder aux pressions les poussant à protéger des entreprises et des emplois en limitant l'émergence d'activités ou de produits concurrents. Or, comme le remarque Amartya Sen, en citant Adam Smith: «restreindre la concurrence dessert toujours l'intérêt public et ne saurait avoir d'autre fin que de permettre au négociant, élevant ses bénéfices au delà de ce que ces derniers auraient naturellement dû être, de lever sur le reste de ces concitoyens, et à son seul bénéfice, une taxe absurde $»^{4}$. Dans ce domaine, ce ne sont d'ailleurs pas les importations de produits étrangers qui sont le plus souvent visées. Les restrictions d'importations sont en effet aujourd'hui limitées par les règles de la concurrence internationale. Bien plus nombreuses sont les réglementations limitant la concurrence dans des secteurs protégés de la concurrence internationale. Sur ce point, nous verrons que la France est dans une situation particulièrement défavorable. Et ceci est à rapprocher de la faiblesse de l'emploi dans les secteurs abrités. En effet, si la France avait le même taux d'emploi ${ }^{5}$ que les Etats-Unis dans le commerce, l'hôtellerie et la restauration ${ }^{6}$, secteurs protégés, en grande partie, de la concurrence internationale, elle aurait 3,4 millions d'emplois supplémentaires ; la même comparaison avec les Pays-Bas aboutit à 1,8 million d'emplois, et à 1,2 million dans les cas de l'Allemagne et du Danemark. De fait, en France, ce n'est pas la concurrence internationale qui est source de la faiblesse de l'emploi', les gisements d'emploi sont dans le secteur tertiaire abrité, et en particulier dans le commerce, l'hôtellerie et la restauration.

Ce constat est à rapprocher des résultats de plusieurs travaux récents selon lesquels l'insuffisance d'emploi en France provient en grande partie d'une réglementation de la concurrence inadaptée qui favorise la constitution de multiples pouvoirs de monopole dans des secteurs abrités de la concurrence internationale. L'inadaptation de la réglementation de la

\footnotetext{
4 A martya Sen, Un nouveau modèle économique, O dile Jacob Poches, 2003, p. 167.

${ }^{5}$ Le taux d'emploi est égal au nombre de personnes en emploi divisé par la population en âge de travailler, qui correspond habituellement aux personnes âgées de 15 à 64 ans.

${ }^{6}$ Ces points sont développés dans le rapport de Pierre Cahuc et Michèle Debonneuil, Productivité et emploi dans les services, Rapport nº 40 du CAE, 2004.

${ }^{7}$ Voir Lionel Fontagné, F aut-il avoir peur des délocalisations ? En temps réels, Cahier n³5, Janvier 2005.
} 
concurrence se traduit par la mise en place de cartels et par des mouvements de concentration des entreprises qui conduisent à la domination des mieux dotés au détriment du bien-être de tous. Les monopoles imposent des prix élevés aux consommateurs et raréfient les produits et l'emploi pour conforter leur domination. Ils imposent aussi des salaires faibles aux travailleurs lorsque ces derniers ne sont pas protégés par des syndicats puissants. De ce point de vue, de nombreux travaux convergent pour montrer que les barrières à la concurrence sont défavorables à l'emploi, à la croissance et au pouvoir d'achat.

Cependant, la concurrence n'est pas une panacée. Elle est génératrice de souffrances, surtout pour les personnes les plus fragiles, susceptibles d'être évincées à tout moment par l'arrivée de rivaux plus efficaces ou plus puissants. La concurrence peut avoir des conséquences désastreuses si elle est mal régulée et si les individus sont imparfaitement assurés, en particulier par le système d'indemnisation du chômage, ou s'ils sont peu ou pas accompagnés dans leur recherche d'un travail par les service publics de l'emploi'. Pour que la concurrence profite à tous, il ne faut pas que les gains qu'elle procure ne bénéficient qu'à quelques uns. Ces gains doivent profiter à l'ensemble du corps social par l'avènement d'un système d'assurance efficace permettant de mutualiser les risques de l'existence, en particulier les risques de perte d'emploi. Ce n'est malheureusement pas encore le cas en France, où l'absence d'un tel système suscite des attitudes de repli, légitimes dans le contexte institutionnel actuel, mais défavorables à l'emploi et au consommateur.

\section{Grandeur et faiblesse de la concurrence}

Pour ses détracteurs, la concurrence est une institution barbare, qui assure le règne des plus forts au détriment des plus faibles. Pour ses avocats, c'est au contraire une institution hautement civilisée : une «main invisible», une source d'émulation réalisant la conciliation des intérêts particuliers et de l'intérêt général. En réalité, ces deux conceptions ne sont pas antinomiques, car la concurrence à la fois une formidable machine à produire des richesses, mais aussi une formidable machine à créer des inégalités.

\section{Concurrence et destrudion créatrice}

En permettant l'éclosion d'activités nouvelles, une économie où règne la concurrence entre les producteurs permet d'accroitre en permanence la taille du gâteau que la société peut se 
partager. Malheureusement ce «miracle » a des contreparties : l'éclosion de nouvelles activités, en créant de nouveaux emplois, plus productifs, détruit des emplois anciens, devenus insuffisamment productifs. Ainsi, dans une économie concurrentielle, les entreprises doivent sans cesse innover, en essayant de nouvelles méthodes de production et de gestion du personnel, ou en tentant de vendre de nouveaux produits, pour améliorer ou simplement mintenir leur rentabilité. Certaines innovations sont fructueuses, d'autres échouent. Les entreprises ayant la capacité et la chance d'adopter les bonnes innovations prennent des parts de marché aux autres. C'est dans ces essais et ces erreurs, motivés par la recherche du profit, que la croissance de la productivité trouve sa source et se solde par des réallocations d'emplois entre les entreprises, mais aussi au sein des entreprises. En ce sens, les destructions d'emplois sont bien l'expression d'une création de valeur supplémentaire: des emplois sont détruits dans une entreprise parce que d'autres, plus productifs, sont créés dans cette entreprise ou ailleurs. Telle est la logique du processus de destruction créatrice cher à Joseph Schumpeter. Selon cette logique, la prospérité provient des réallocations d'emplois.

L'ampleur de ce processus de création et de destruction d'emplois est telle qu'un pays comme la France détruit chaque jour ouvrable 10000 emplois. Mais il ne faut pas oublier que ces destructions d'emplois sont en écrasante majorité la conséquence de créations d'emplois, plus productifs, permettant de produire à moindre coût et donc d'obtenir des produits moins chers, avec des salaires et des profits plus élevés. La nature des réallocations d'emploi est souvent méconnue car ses bienfaits, contrairement à ses inconvénients, sont diffus et peu apparents. En effet, si les destructions d'emploi induites par la concurrence sont apparentes et sans cesse dénoncées, les créations d'emplois, qui sont aussi possibles grâce au jeu de la concurrence, sont peu médiatisées, car elles sont considérées comme un phénomène normal. On a donc tendance à les oublier. En témoignent l'audience et la mansuétude accordées à quelques apôtres de la disparition inéluctable du travail. Lorsqu'en 1996, Viviane Forrester proclame, dans son livre L 'horreur éonomique, que les termes de «créations d'emplois » sont une «formule que l'on sait vide, définitivement flétrie, mais qui n'en est pas moins incontournable, car cesser de mentir à œ propos pourrait vite signifier oesser d'y croire, avoir à se réveiller pour se déouvvir au sein d'un cauchemar qui n'appartient pas au domaine du sommeil, ni même du rêve éveillé $\iota^{9}$, elle ne considère à l'évidence que les destructions et oublie les créations. Jeremy Rifkin fait preuve du même aveuglement dans son livre La fin du travail, paru en 1995 aux Etats-Unis et qui fut un autre grand succès de librairie. Il écrit un livre de plus de 400 pages, étayées d'une multitude d'exemples d'entreprises détruisant des emplois, pour

${ }^{8}$ La concurrence peut en effet entraîner des transformations du système productif dont les conséquences sont très néfastes pour de larges couches de la société. L'ouvrage de Karl Polanyi, L a G rande transformation, Gallimard, 1983, décrit ce phénomène durant la période de la révolution industrielle en $\mathrm{G}$ rande Bretagne. 
« démontrer» que l'heure de la fin du travail a sonné. Si la France détruit à elle seule 10000 emplois par jour, il n'est pas difficile de raconter des dizaines d'histoires de faillites ou de compression de personnel.

Les économies industrialisées détruisent certes beaucoup d'emplois, mais elles en créent aussi beaucoup et, ironie de l'histoire, au cours des cinq années qui suivirent la parution de ces deux livres, les créations d'emplois dépassèrent sensiblement les destructions, non seulement aux Etats-Unis, ce qui est habituel, mais aussi en France. Toutes les données dont nous disposons n'apportent pas le moindre début de preuve à ces pseudo théories catastrophistes. Comment expliquer alors l'audience et le succès de ces faux prophètes ? Il est probable que l'évocation de l'apocalypse frappe immédiatement et sans effort l'imagination. Face à cela, la présentation d'un bilan nuancé prend vite l'allure d'un pensum ennuyeux et, par nature, contestable. De plus, les destructions d'emplois sont souvent synonymes de drames personnels. La détresse et la colère peuvent être facilement instrumentalisées, tandis que les créations d'emplois sont le plus souvent diffuses et, en règle générale, il n'y a rien à montrer. Jouer sur les peurs millénaristes (et celle de la fin du travail en fait partie) aura toujours plus d'écho qu'une étude sérieuse, chiffrée qui aboutit à des conclusions peu spectaculaires et pas toujours tranchées.

D estruction créatriœe, emploi et productivité

Disposant aujourd'hui de nombreuses données issues d'enquêtes couvrant de longues périodes, les études récentes montrent que les innovations améliorent la productivité principalement grâce au processus de destruction créatrice. Pour une part importante ce processus a lieu au sein des entreprises existantes ${ }^{10}$, mais c'est loin d'être toujours le cas. La conjugaison des réallocations d'emplois entre des entreprises différentes, l'apparition de nouvelles entreprises et les disparitions d'entreprises plus anciennes expliquent la moitié de la croissance de la productivité du secteur manufacturier nord américain dans les années 1980 et $1990^{11}$. Les chiffres sont encore plus frappants dans le secteur du commerce de détail. D epuis la fin des années 1980, celui-ci a connu une révolution liée au progrès de l'informatique. Cette révolution tient à l'usage

\footnotetext{
9 D ans L 'horreur éonomique, F ayard, 1996, page 74.

10 Pour une étude portant sur dix pays de l'OCDE, voir Stefano Scarpetta, Phillip Hemmings, Thierry Tressel et Jaejon Woo, "The role of policy and institution for productivity and firm dynamics: evidence from micro and industry data", Working Paper $\mathrm{n}^{\circ} 329,2002$, OECD Economics Department. Disponible à l'adresse www.oecd.org/ eco.

11 Voir Lucia Foster, John Haltiwanger, et C.-J Krisan, "Aggregate productivity growth: lessons from microeconomic evidence » dans D ean, E., Harper, M. et Hulten, C. (éditeurs), N ew D evelopments in Productivity A nalysis, University of Chicago Press, 2001. Cette étude montre aussi que dans le sous-secteur de la réparation automobile, la croissance de la productivité est entièrement due aux nouvelles firmes de ce sous secteur et, qu'en moyenne, les anciennes firmes auraient eu tendance à faire baisser la productivité.
} 
des codes barres, mais aussi à la transmission immédiate de tous les achats enregistrés aux caisses vers les services d'approvisionnement qui peuvent à leur tour ajuster très rapidement l'état des stocks. Aux Etats-Unis, sur la décennie 1987-1997, les réallocations entre entreprises comptent pour plus de $80 \%$ de la croissance de la productivité du secteur. En d'autres termes, moins de $20 \%$ de la croissance de la productivité du commerce de détail proviennent des réorganisations au sein d'un même établissement ${ }^{12}$.

Pourquoi les réorganisations des emplois au sein des établissements existants n'ont-elles, dans certains secteurs, qu'une responsabilité modeste dans la croissance de la productivité ? Ricardo Caballero et Mohamad Hammour ${ }^{13}$ ont particulièrement étudié cette question. Leur principale conclusion est que le fonctionnement d'une entreprise a toujours un fort degré de spécificité Chaque entreprise est une alchimie complexe, façonnée par le temps, de savoir faire technique, d'imbrications de liens personnels ou collectifs pas toujours explicites, d'organisation du travail ayant ses règles et ses coutumes, de manière de prendre des décisions, etc. Cet amalgame est en grande partie propre à chaque entreprise et il ne fonctionne bien qu'au sein de cette entité particulière. Une entreprise ressemble plus à un système composé de liaisons irréversibles entre certains de ses éléments qu'à une collection d'individus indifférenciés et de machines que l'on pourrait remodeler à l'envi. Lorsque apparait une innovation technologique ou lorsque les conditions de la concurrence se modifient, certaines entreprises ou certaines unités d'une même entreprise ne sont pas capables de s'y adapter, car le degré de spécificité des relations internes est trop fort. Elles disparaissent, en partie ou en totalité, et en même temps des entreprises ou des établissements mieux adaptés émergent, au profit du plus grand nombre. La profonde réorganisation du transport aérien au début $d u 21^{\text {ième }}$ siècle illustre à merveille ce constat.

La croissance de la productivité fait pourtant peur. En effet, chacun a en tête un mécanisme a priori logique : pour améliorer la productivité, il faut licencier. En fait, les études chiffrées démontrent exactement le contraire. Par exemple, Bruno Crépon et Richard Duhautois trouvent, qu'en France, au cours des années 80 comme au cours des années 90, ce sont les entreprise dont la productivité croît le plus vite qui créent le plus d'emploi. Une étude portant sur les Etats-Unis aboutit à un constat similaire ${ }^{14}$. Ainsi, contrairement à la croyance populaire, il apparait qu'augmenter la productivité est, en moyenne, favorable à l'emploi.

\footnotetext{
12 Voir Lucia Foster, John Haltiwanger, et C.-J Krisan, «The link between aggregate and micro productivity growth : evidence from retail trade», NBER Working Paper $\mathrm{n}^{\circ}$ 9120, août 2002. Disponible à l'adresse http:/ / www.nber.org/ papers/ w9120.

13 En particulier dans leur article, «On the timing and efficiency of creative destruction», Q uarterly Journal of E conomics, vol 111, pp. 805-852.

14 Baily, M., Bartelsman, E. et Haltiwanger, J., « Labor Productivity: Structural Change and Cyclical Dynamics », The Review of $\mathrm{E}$ conomics and Statistics, 2001, vol. 83, n 3, pp. 420-433.
} 
Comment la concurrence profite à tout le monde... aux détriments de certains : l'ex emple du transport aérien

Les attentats du 11 septembre 2001 ont précipité la dégradation du secteur du transport aérien. La plupart des grandes compagnies ont réduit leurs flottes, d'autres (comme Swissair ou Sabena) ont simplement disparu. Pourtant, entre l'été 2001 et l'été 2002, les six plus grands transporteurs «à bas coûts » (Ryanair, Easyjet, Buzz, Virgin Express, Go et Bmibaby) ont, en moyenne, augmenté leur nombre de passagers de $48 \%$. La réussite de ces compagnies ne s'explique pas uniquement par leur «bas coûts »; elles ont aussi introduit quelques innovations Elles ont accru le nombre de sièges par appareil, ne fournissent aucun service à bord, n'entretiennent pas de grands réseaux, possèdent souvent un seul type d'appareil, ont des structures administratives minimales, utilisent souvent de petits aéroports délaissés par les transporteurs traditionnels, etc. La somme de ces innovations aboutit à ce que la productivité du travail dans une compagnie «à bas coûts» dépasse nettement celle d'un transporteur traditionnel. Ainsi, en avril 2002, il y avait en moyenne 227 employés par appareil chez Air France, 254 chez British Airways, mais ces chiffres tombent à 76 chez Virgin Express, à 68 chez Easyjet et à 36 chez Ryanair, sans aucun impact, notons le, sur la sécurité des passagers. Les compagnies «à bas coûts » ont créé des emplois (chez elles et dans tout le secteur du transport aérien) tandis que les compagnies traditionnelles en ont détruits. Par exemple, en 2001, en Europe, 30\% des embauches de pilotes ont été réalisées par les transporteurs «à bas coûts » alors que ces derniers représentent, à cette date, à peine $11 \%$ du trafic passager européen ${ }^{15}$.

Le processus de destruction créatrice s'est réalisé au bénéfice des compagnies à «bas coûts » et au détriment des transporteurs traditionnels. Pourtant ces demiers ont tenté de réagir, en créant parfois leur propre filiale «à bas coûts », mais avec un succès relatif. Les experts du secteur estiment d'ailleurs que les transporteurs traditionnels ne parviendront pas véritablement à concurrencer les compagnies «à bas coûts», car le degré de spécificité de l'organisation d'une compagnie traditionnelle est trop fort pour qu'elle devienne, ou donne naissance, à une compagnie «à bas coûts». Comme le suggère avec humour Leo Mullin, le patron de Delta Airlines la troisième compagnie américaine, «le bébé d'un dinosaure sera toujours un dinosaure $»^{16}$. Au bout du compte, les compagnies à «bas coûts » auront fait considérablement progresser la productivité du secteur du transport aérien. Et, sauf nouvelle catastrophe, de plus en plus de gens prendront l'avion (car il devient en moyenne moins cher), de plus en plus de nouvelles lignes

\footnotetext{
15 Toutes ces données se trouvent sur le site www.eca-cokpit.com.

${ }^{16}$ Ces propos et d'autres données se trouvent dans l'article «Les compagnies à bas prix déferlent sur l'Europe» paru dans le journal L e M onde du 12 décembre 2002.
} 
seront ouvertes (car des destinations non rentables avec les transporteurs traditionnels le deviennent avec les compagnies «à bas coûts ») et les emplois auront été redistribués au sein du secteur du transport aérien ${ }^{17}$. Le processus de destruction créatrice aura accompli sa tâche.

L'exemple du transport aérien n'est pas isolé. Tous les pays de l'O CDE sont affectés par un fort taux de création et de disparition d'entreprises. Eric Bartelsman, Stefano Scarpetta et Fabiano Schivardi ${ }^{18}$ ont constaté qu'environ $10 \%$ des entreprises disparaissent en moyenne, chaque année, dans chaque pays de l'O CDE. Bien évidemment, comme pour les destructions d'emplois, les destructions d'entreprises sont généralement compensées par des créations : chaque année le nombre d'entreprises créées représente 10\% des entreprises existantes.

L es ennemis de la concurrence

Dans de nombreuses circonstances, les acteurs du jeu politique peuvent retirer des bénéfices substantiels à protéger les victimes potentielles de la concurrence en la limitant, voire en l'empêchant, par l'intermédiaire d'une réglementation adaptée. Telle est la stratégie adoptée par Georges Bush, qui choisit de restreindre les importations d'acier pour protéger la sidérurgie traditionnelle dans l'Etat de Virginie O ccidentale au début des années 2000. En l'occurrence, Georges Bush avait raison, puisque ce choix a contribué à lui faire gagner les élections dans cet Etat ! Néanmoins, ce choix a aussi eu pour contrepartie de réduire le volume total d'emploi aux Etats-Unis dans la mesure où le nombre d'emplois conservés grâce aux barrières douanières était nettement inférieur à ceux détruits par ces mêmes barrières. Il faut malgré tout reconnaître, qu'en la matière, G eorges Bush est loin d'avoir innové. L'histoire pullule d'exemples dans lesquels des groupes d'intérêts parviennent à protéger leurs activités en limitant la concurrence grâce au pouvoir politique. Et les plus farouches ennemis de la concurrence ne sont pas toujours ceux que l'ont croit. En effet, les travailleurs susceptibles de perdre leur emploi ne sont pas les seuls à être opposés à la concurrence. Ses plus farouches opposants sont fréquemment des «capitalistes »à la tête de très grandes entreprises, cherchant à préserver ou à gagner des parts de marché par des procédés qui peuvent relever de la simple entente, du lobbying ou de manipulations idéologiques sophistiquées. Comme le souligne Fernand Braudel, cette tendance, très ancienne, rythme la

\footnotetext{
17 Un accroissement sectoriel de la productivité du travail entrâne des gains d'emploi si la demande pour le produit du secteur est sensible au prix (plus exactement, si l'élasticité prix de la demande du produit est supérieure à l'unité). En effet, dans ce cas, les diminutions de prix induites par les gains de productivité conduisent à accroître fortement la demande, ce qui est favorable à l'emploi. Dans le cas contraire, les gains de productivité entrânent une diminution sectorielle de l'emploi. par un accroissement de l'emploi dans les secteurs où la demande est sensible au prix.

18 "Comparative analysis of firm demographics and survival: micro-level evidence for the OECD countries" Document de recherche du département d'économie de l'OCDE n³48, 2003; disponible gratuitement sur http:/ / ideas.repec.org/ p/ oecd/ oecdec/ 348.html.
} 
dynamique du capitalisme depuis son émergence : «Que ces capitalistes, en Islam comme en Chrétienté, soient les amis du prince, des alliés ou des exploiteurs de l’Etat, est-il besoin de le dire ? Très tôt, depuis toujours, ils dépassent les limites «nationales», s'entendent avec les marchands des places étrangères. Ils ont mille moyens de fausser le jeu en leur faveur, par le maniement du crédit, par le jeu fructueux des bonnes contre les mauvaises monnaies... ils ont la supériorité de l'information, de l'intelligence et de la culture. Et ils saisissent toujours autour d'eux ce qui est bon à prendre. Q u'ils aient à leur disposition des monopoles ou simplement la puissance nécessaire pour effacer neuf fois sur dix la concurrence, qui en douterait ? E crivant à l'un de ses comparses de Bordeaux, un marchand hollandais lui recommandait de tenir secrets leurs projets; autrement, ajoutait-il, «il en serait de cette affaire comme de tant d'autres où, dès qu'il y a de la concurrence, il n'y a plus d'eau à boire $» ! »^{19}$.

Ainsi, Braudel souligne, tout au long de son ouvrage consacré à la dynamique du capitalisme que le principal ennemi de la concurrence est bien le capitaliste. Il conclut son analyse en déclarant : «Ce que je regrette pour ma part, non en tant qu'historien, mais en tant qu'homme de mon temps, c'est que dans le monde capitaliste comme dans le monde socialiste, on refuse de distinguer capitalisme et économie de marché. A ceux qui, en Occident, s’attaquent aux méfaits du capitalisme, les hommes politiques et les économistes répondent que c'est là un moindre mal, l'envers obligatoire de la libre entreprise et de l'économie de marché. Je n'en crois rien. A ceux qui, selon un mouvement sensible même en URSS, s'inquiètent de la lourdeur de l'économie socialiste et voudraient lui ménager plus de «spontanéité » (je traduirais : plus de liberté), la réponse est que c'est la un moindre mal, l'envers obligé de la création du fléau capitaliste. Je n'en crois rien non plus. Mais la société qui serait pour moi idéale est-elle possible ?Je ne pense pas en tout cas qu'elle ait beaucoup de partisans à travers le monde ! » ${ }^{20}$.

\section{L a fragilité des économies de marché}

L'économie de marché, au sens où l'entend Fernand Braudel, est un système très fragile et menacé en permanence car, si la concurrence bénéficie au plus grand nombre, les pertes qu'elle peut occasionner à quelques uns ou à quelques groupes sont, pour ceux-là, infiniment plus grandes que les gains des autres. A titre individuel, personne ne fait d'énormes profits à défendre la concurrence, ce qui limite fortement la formation de coalitions en faveur de la concurrence. A l'inverse, les perdants, comparativement peu nombreux mais subissant de fortes pertes, ont au contraire de fortes incitations à se coaliser. Concrètement, les perdants du jeu concurrentiel, qu'ils

\footnotetext{
${ }_{19}$ Fernand Braudel, L a dynamique du capitalisme, Arthaud, 1985, pp. 60-61.
} 
soient salariés ou entrepreneurs, seront les seuls à faire pression sur les pouvoirs publics pour exiger des restrictions à la concurrence. Le comportement de Georges Bush en Virginie O ccidentale aux élections présidentielles de 2000 n'est qu'une illustration somme toute banale d'une absurdité économique dans la mesure où le coût d'une politique d'indemnisation et d'aide aux reclassement est généralement bien inférieur à l'amputation du pouvoir d'achat des consommateurs consécutive à l'instauration des barrières tarifaires. Toute aussi banale est la menace de rétablissement des quotas textiles brandie, au début de l'année 2005, par l'Union européenne (et les Etats-Unis) pour faire face à «l'invasion » des produits chinois et indiens. Sur ce point, David Spector, un économiste chercheur au CNRS, s'est livré à un petit calcul très instructif. En admettant que ladite «invasion » fasse baisser les prix des textiles de $5 \%$, ce qui est une hypothèse basse, le transfert de pouvoir d'achat au bénéfice des ménages français serait de l'ordre de 1,5 milliard d'euros par an. En revanche, si l'Etat décidait de payer aux 7000 personnes menacées en 2005 par la fin des quotas (c'est le chiffre avancé par Patrick Devedjian, le ministre de l'Industrie de l'époque), leurs salaires jusqu'à leur retraite et de verser les cotisations sociales correspondantes aux organismes adéquats, il lui en coûterait environ 200 millions d'euros par an, soit approximativement 7 fois moins que les gains en termes de pouvoir d'achat ${ }^{21}$ !

L'instauration de quotas limitant les importations ne constitue qu'une forme parmi d'autres de réglementation anticoncurrentielle. De telles réglementations, qui protègent des innovations tout autant que de la concurrence internationale, sont très fréquentes, en particulier en France, où elles exercent un impact négatif important sur l'emploi et le pouvoir d'achat.

\section{Le poids des réglementations anticoncurrentielles}

A priori, pour lutter contre les destructions d'emplois, il peut être tentant de protéger les entreprises en place par une réglementation limitant l'entrée sur le marché de nouvelles firmes utilisant des technologies différentes et moins intensives en main-d'œuvre. L'institution de telles barrières permet de réduire les destructions d'emplois à court terme. A ce titre, elles peuvent être favorables à l'emploi. Néanmoins, étant donnée l'ampleur des rotations d'emploi, les effets de court terme s'estompent très vite. Rapidement, les barrières à l'entrée ont pour effet essentiel d'exercer une pression à la hausse sur les prix, ce qui est toujours défavorable à l'emploi. Elles ont aussi tendance à limiter les innovations, ce qui freine l'apparition de nouveaux produits et est aussi généralement défavorable à l'emploi. Les barrières à l'entrée contribuent enfin à diminuer les gains de productivité, ce qui peut être bénéfique à l'emploi du secteur si la demande pour le

\footnotetext{
20 Ibid, pp. 118-119.

${ }^{21}$ Voir l'article paru dans le journal L ibération du 6 juin 2005 sous le titre « Textile chinois, le bon marché».
} 
produit concerné est faiblement sensible au prix ${ }^{22}$. L'impact des barrières à l'entrée sur l'emploi d'un secteur et sur l'emploi global est donc ambigu en théorie ; il ne peut être connu que grâce à une exploration empirique. Le consommateur, pour sa part, subit toujours une diminution de bien-être puisqu'il paie les produits plus chers et ne bénéficie pas des innovations de produits.

Les études empiriques disponibles montrent que les régulations anticoncurrentielles, dont le but est de protéger les emplois existants en limitant l'entrée d'entreprises utilisant des technologies nouvelles, se traduisent par des pertes d'emploi globales. En France, deux cas d'école illustrent ce propos. Celui que nous envisagerons en premier porte sur la réglementation du commerce et de l'artisanat, qui restreint, notamment, l'installation de magasins de grandes surfaces depuis 1973. Le second concerne une modification de la réglementation du transport routier intervenue en 1986, qui a supprimé l'autorisation administrative jusqu'alors nécessaire pour transporter des marchandises au-delà de 150 kilomètres. Ces deux exemples ne recouvrent qu'une infime partie des secteurs et des professions réglementées. Les études microéconomiques, analysant de façon détaillée l'impact des réglementations sur l'emploi et les consommateurs, manquent en effet cruellement. Il ne peut donc s'agir, en aucune manière, de condamner en bloc l'efficacité de toutes les réglementations qui limitent l'entrée. Chacune d'entre elles doit être examinée précisément avant d'être éventuellement modifiée. Néanmoins, les études macroéconomiques, réalisées grâce à des comparaisons internationales, indiquent que les réglementations anticoncurrentielles, particulièrement nombreuses en France, ont un impact significativement négatif sur l'emploi. En outre, ces comparaisons internationales montrent que la prolifération de réglementations anticoncurrentielles a tendance à accentuer la corruption, car elle favorise les pressions politiques et le lobbying.

L e commerœe de détail en F ranœ

La loi d'orientation du commerce et de l'artisanat du 27 décembre 1973, dite loi Royer, a fait l'objet récemment de nombreuses critiques et d'autant de propositions de réformes ${ }^{23}$. A l'origine, ce dispositif était supposé protéger les petits commerces, dans une période où la grande distribution se développait. Pour justifier cette loi, les auteurs des textes invoquaient «la nécessité d'assurer un développement équilibré de toutes les formes de commerce en prévenant les risques de dévitalisation des centres-villes et de désertification des zones rurales ». Le dispositif central de

\footnotetext{
22 Plus précisément, tel est le cas si l'élasticité de la demande par rapport au prix est inférieure à un.

23 Voir en particulier le «rapport du groupe d'ex perts onstitué sur les rapports entre industrie et commerce», présidé par M. Guy Canivet pour le compte du ministre de l'économie, des finances et de l'industrie, 18 octobre 2004, et le rapport «Pour une nouvelle croissance française » présenté par un autre groupe d'experts présidé par M. Michel Camdessus et présenté aussi au ministre de l'économie, des finances et de l'industrie en septembre 2004.
} 
la loi Royer est une procédure d'autorisation préalable à l'ouverture de toute grande surface. En d'autres termes, la loi Royer prévoit d'instaurer le cas échéant des barrières à l'entrée des grandes surfaces dans le but avoué de protéger le petit commerce. En pratique, jusqu'en 1996, toute création ou extension d'un magasin de vente au détail devait faire l'objet d'une demande d'autorisation lorsque sa surface dépassait $1000 \mathrm{~m}^{2}$ dans une commune de moins de 40000 habitants et $1500 \mathrm{~m}^{2}$ pour une commune de plus de 40000 habitants. En 1996, le dispositif a été renforcé et concerne désormais toute surface de vente de plus de $300 \mathrm{~m}^{2}$. La demande d'autorisation se fait auprès d'une «Commission départementale d'équipement commerciaux » composée d'élus locaux, de représentants consulaires et de représentants des associations de consommateurs.

Un article publié par Marianne Bertrand et Francis Kramarz" a tenté d'évaluer l'impact de la loi Royer sur l'emploi mais aussi sur la concentration et les prix du secteur du commerce de détail, alimentaire et non-alimentaire, en France. Pour la période 1974-1998, les auteurs ont pu dresser la liste exhaustive des demandes d'ouverture et des résultats du vote des commissions locales concernées par ces demandes. On peut ainsi disposer d'une mesure de l'intensité des restrictions à l'entrée propre à chaque localité. Mais il ne suffit pas de mettre en correspondance cette mesure locale avec un indicateur portant sur les créations ou les destructions d'emplois locales pour conclure à une causalité allant de l'existence de barrières à l'entrée vers le niveau de l'emploi. Il est en effet possible que les demandes d'ouverture de grandes surfaces - et donc l'intensité des restrictions à l'entrée -- dépendent du dynamisme économique local. D ans ce cas, une valeur plus ou moins grande des créations d'emplois reflète le plus ou moins grand dynamisme des conditions économiques locales et n'est pas lié à une plus ou moins grande intensité des barrières à l'entrée. Pour éliminer cette éventualité, les auteurs ont constaté que les élus locaux jouent un rôle pivot dans les votes des commissions départementales alors, qu'en règle générale, les représentants des commençants s'opposent aux demandes d'ouvertures, tandis que les représentants des consommateurs y sont favorables. Ainsi, les estimations montrent que plus d'ouvertures sont accordées dans les départements où les élus de gauche, plus proches des associations de consommateurs, sont plus présents. Inversement, moins d'ouvertures ont lieu dans des départements où les élus de droite, plus proches des commerçants, sont mieux implantés. Cette différenciation par la couleur politique permet d'identifier l'effet causal des restrictions à l'entrée sur l'emploi. Si un département de gauche a, toute chose égale par ailleurs, accordé moins d'autorisations d'ouverture, c'est que vraisemblablement la situation économique

\footnotetext{
24 Marianne Bertrand et Francis Kramarz, «D oes Entry Regulation Hinder Job Creation ? Evidence from the French
} Retail Industry », Q uarterly Journal of E conomics, CXVII, 4, 2002, pp. 1369-1414. 
locale est moins bonne et l'on doit éliminer cette influence pour connaitre le véritable effet des barrières à l'entrée sur le niveau de l'emploi.

Cet article aboutit a une conclusion dépourvue d'ambiguité : les départements où les restrictions à l'entrée ont été les plus fortes sont aussi ceux où la création d'emplois dans le secteur du commerce de détail a été la plus faible. Les résultats économétriques suggèrent que l'emploi global dans ce secteur, petites et grandes surfaces confondues, serait aujourd'hui d'au moins 10\% supérieur en l'absence de restrictions ${ }^{25}$. La loi Royer a bien protégé les petits commerces : là où les restrictions sont les plus fortes, leur disparition a été ralentie. Mais, la loi a aussi limité la croissance de l'emploi dans le grand commerce. Au total, l'effet de la loi est négatif. Les estimations économétriques montrent aussi que les restrictions à l'entrée accroissent la concentration locale des grandes chaînes de supermarchés alimentaires ${ }^{26}$. En substance, cela signifie que la plus grande chaîne d'un département (relativement à ses concurrentes) est encore plus grande si les barrières à l'entrée augmentent. Ces restrictions accroissent aussi localement les prix de détail sans que la qualité des biens se modifie. Toutefois, il est peu vraisemblable que cette hausse des prix soit un facteur déterminant dans la mauvaise performance de l'emploi causée par les restrictions à l'entrée car cela impliquerait une sensibilité de la demande aux prix trop élevée $e^{27}$. C'est donc la concentration en soi qui a un effet direct sur la croissance de l'emploi (effet qui s'ajoute à celui des restrictions). La concentration freine la différenciation entre les chaines de supermarchés et la faible différenciation explique en partie les piètres performances en matière d'emploi. Ainsi, aux Etats-Unis, on trouve des chaines «haute-qualité » qui utilisent beaucoup de main-d'œuvre et des chaînes «basse-qualité » qui en utilisent moins. En France, la différenciation reste très faible et n'est due qu'à l'entrée récente d'entreprises d'origine allemande. Il y a peu de temps, les dirigeants d'une de nos plus grandes chaînes ont déclaré s'être rendus compte que certains de leurs clients habitant la Saint-D enis dans la région parisienne, étaient (relativement) pauvres et donc que le format classique de la grande surface indifférenciée n'était pas adapté à cette localité. La différenciation - des supermarchés différents pour des clientèles différentes présente dans de nombreux pays a mis 30 ans pour atteindre la France. De plus, le «manque »de supermarchés rend les trajets des clients plus longs et l'attente, aux caisses par exemple, plus

\footnotetext{
$25 \mathrm{On}$ est encore plus convaincu de la validité de cette conclusion si l'on remarque que la croissance de l'emploi du secteur des hôtels, cafés et restaurants -- secteur proche du commerce de détail par sa structure d'emploi où de nombreux travailleurs à bas salaire sont présents --, n'est nullement affectée par les restrictions à l'entrée dans le commerce de détail du même département. Alors que l'emploi a crû respectivement de $0.6 \%$ et $0.8 \%$ par an avant la mise en place de la loi Royer, dans le secteur du commerce de détail et dans œlui des hôtels, cafés et restaurants, il a crû de $0.1 \%$ et $1.7 \%$ par an dans ces mêmes secteurs après la mise en place de la loi Royer.

${ }^{26}$ Cette concentration est mesurée à chaque date et dans chaque département par la concentration des chiffres d'affaires des entreprises (indice d'Herfindahl), par la part de la surface détenue par la plus grande chaîne, ou par la part de la surface détenue par les deux plus grandes chaînes.
} 
grande. Le consommateur français passe plus de temps que son homologue américain à se rendre à son supermarché et à y faire la queue. La concurrence est autant l'alliée de l'emploi que des consommateurs.

Sucoès et ratés de la dérégulation du transport routier de marchandises

En 1986, la réglementation du transport routier de fret a été modifiée en France. Auparavant, il existait un système de licence ayant pour but de protéger le chemin de fer de la concurrence du transport routier. Chaque camion roulant (environ) plus de 150 kilomètres devait obligatoirement posséder une licence, délivrée par l'Etat en nombre limité. En outre, la «tarification routière obligatoire » définissait des planchers permettant de limiter la baisse des tarifs. En 1986, les licences furent distribuées très largement et la tarification obligatoire fut supprimée. Comme le souligne Emile Quinet, «Les exigences de flexibilité, le besoin de développer des structures bénéficiant d'effets de réseaux, ont entrâné une augmentation de leur taille: le nombre total d'entreprises augmentait de plus de $10 \%$ en raison des possibilités nouvelles ouvertes à l'entrée sur le marché. ${ }^{28}$. Il est facile de comprendre ce qui a permis ce développement à l'aide des quatre graphiques qui suivent.

27 Toutefois, l'élasticité des prix aux restrictions d'entrée estimée est environ trois fois plus petite que l'élasticité de l'emploi aux restrictions d'entrée.

28 Voir sur tous ces points et d'autres, Emile Quinet, «Les politiques de libéralisation des transports : une analyse comparative», Problèmes E conomiques, 1999, novembre. 


\section{Prix Relatif du Secteur des Transports}

Source: Comptes Nationaux

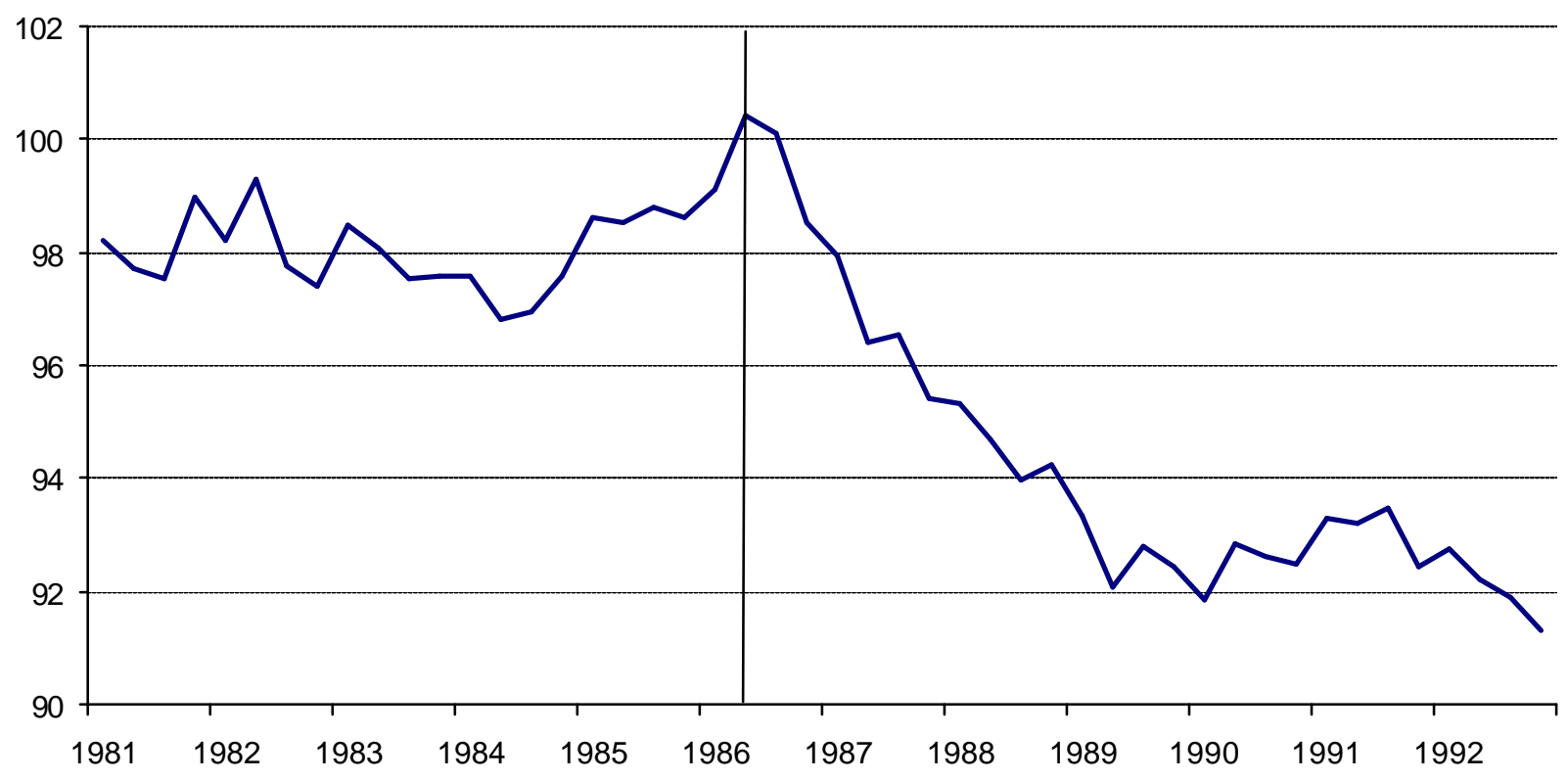

Figure 1: Evolution du prix relatif des prestations du secteur des transports entre 1981 et 1993. Source : Comptes Nationaux.

La figure 1 indique clairement que le prix relatif des prestations du secteur des transports baisse massivement dès 1986. Les travaux récents de Pierre-Philippe Combes et Mirène Lafourcade ${ }^{29}$ démontrent que la baisse des coûts qui eut lieu à cette période est essentiellement due à cette politique de «ibéralisation». Et cette baisse, intensifiée par la concurrence, s'est traduite par une baisse des profits qui, avant la libéralisation, étaient plus élevés que ceux des autres secteurs et qui ont baissé tout de suite après 1986, comme l'indique la figure 2 suivante.

29 Pierre Philippe Combes et Miren Lafourcade, «Transport Costs: Measures, D eterminants, and Regional Policy Implications for France », 2004, document de travail CERAS, 04- 05,

http:/ / www.enpc.fr/ ceras/ labo/ documents.html. 


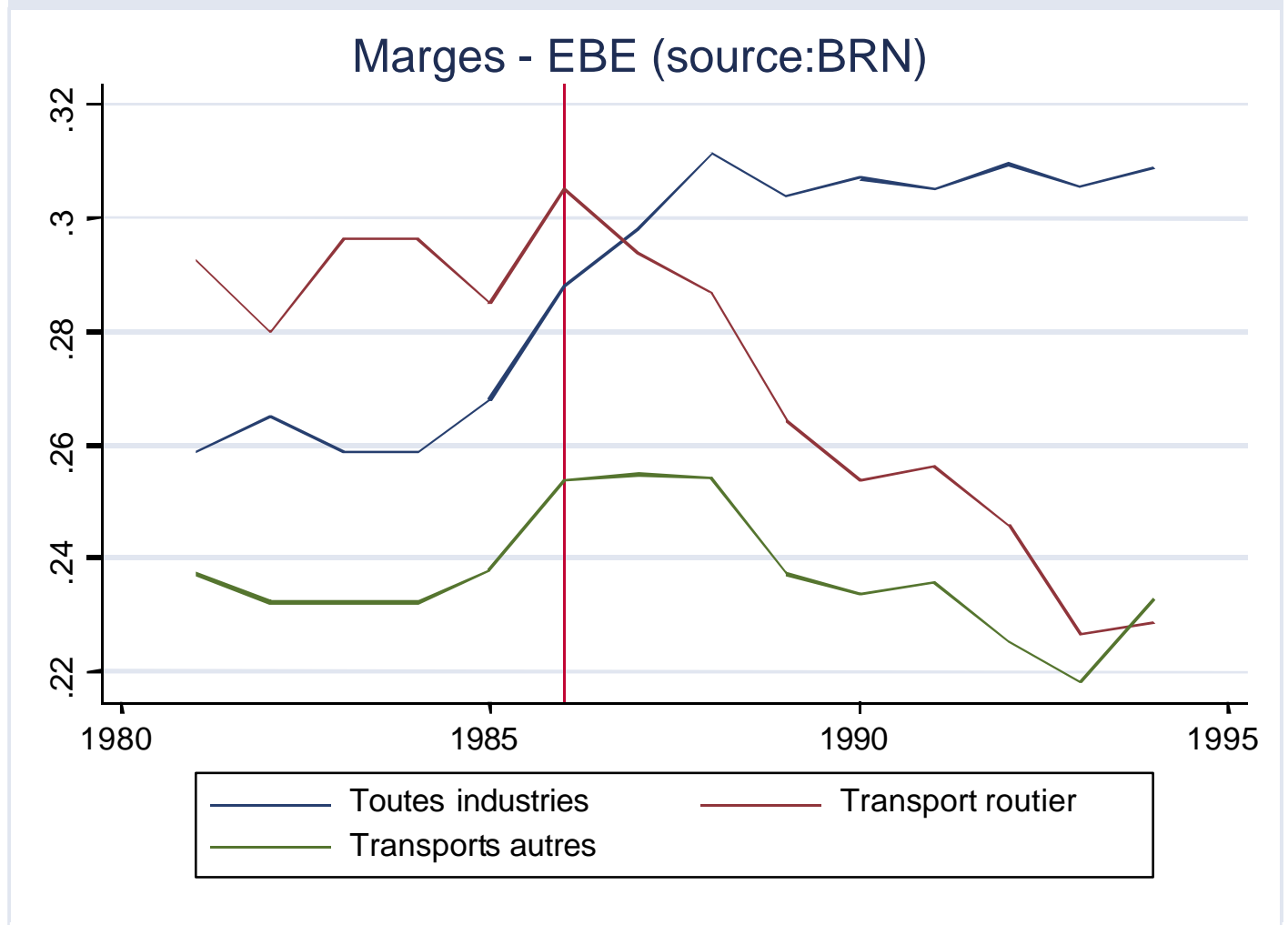

Figure 2: Evolution des taux de marge dans le secteur des transports et dans l'industrie entre 1980 et 1995. Source : BRN.

Très logiquement, la figure 3 montre que l'activité dans le secteur du transport de fret routier augmente massivement et quasiment simultanément à la libéralisation. Sans surprise l'emploi dans ce secteur a explosé puisqu'il croissait au rythme de 1.5\% dans les années précédant 1986, et s'est mis à augmenter de plus de 5\% par an de 1986 à 1990. Un léger ralentissement suivit cette période, mais depuis 1994 le rythme de croissance de l'emploi est proche de 4\%. Au total, sur la période l'emploi a été multiplié par deux, passant de 170000 en 1976 à 340000 en 2001 comme le montre le graphique 4. 


\section{Activité dans le Transports de Fret Routier}

Source: Eurostat

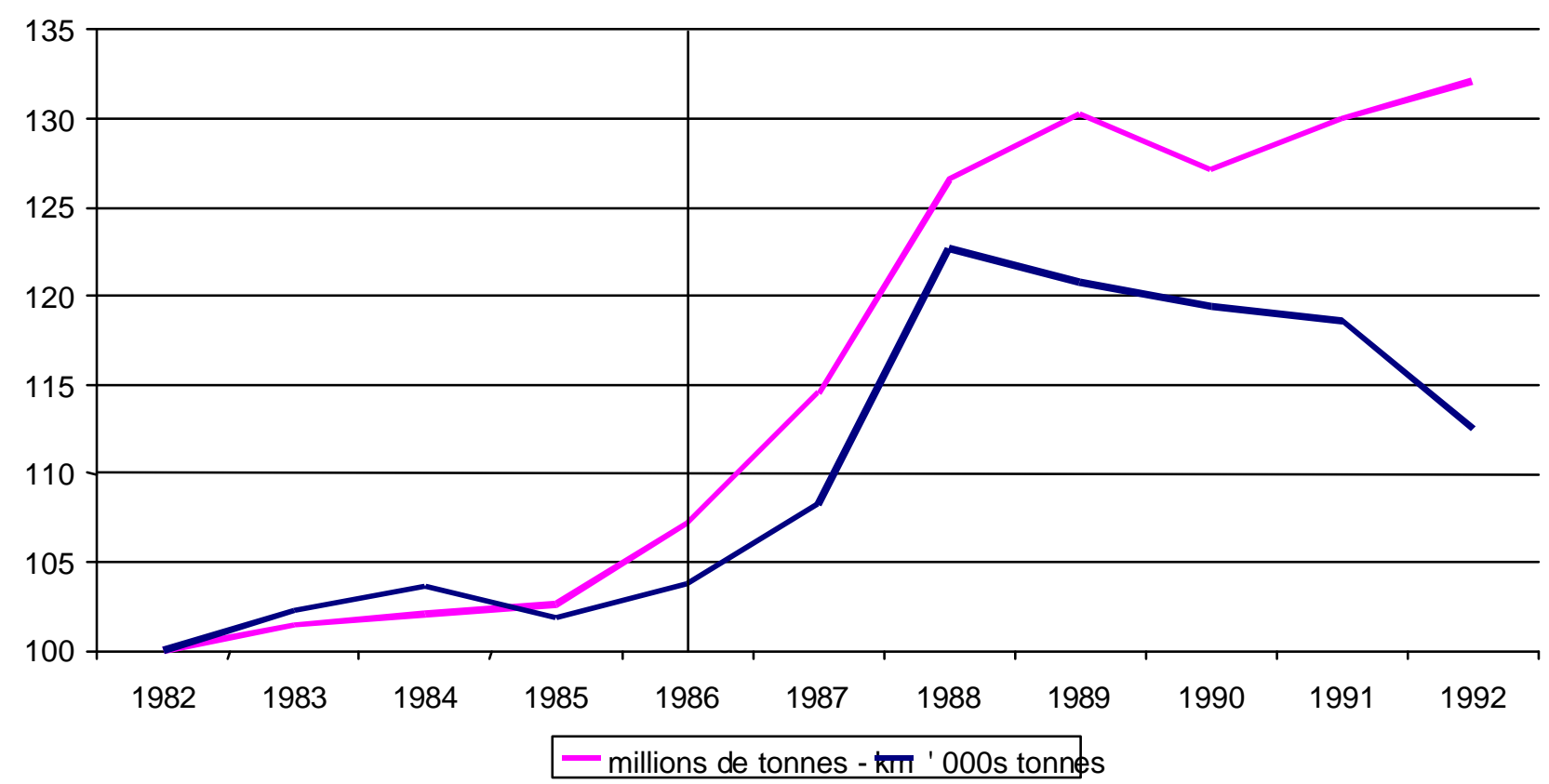

Figure 3 : Evolution de l'activité dans le secteur des transport de fret routier et dans l'industrie entre 1982 et 1993. Source : E UROSTAT.

Transport routier de marchandises

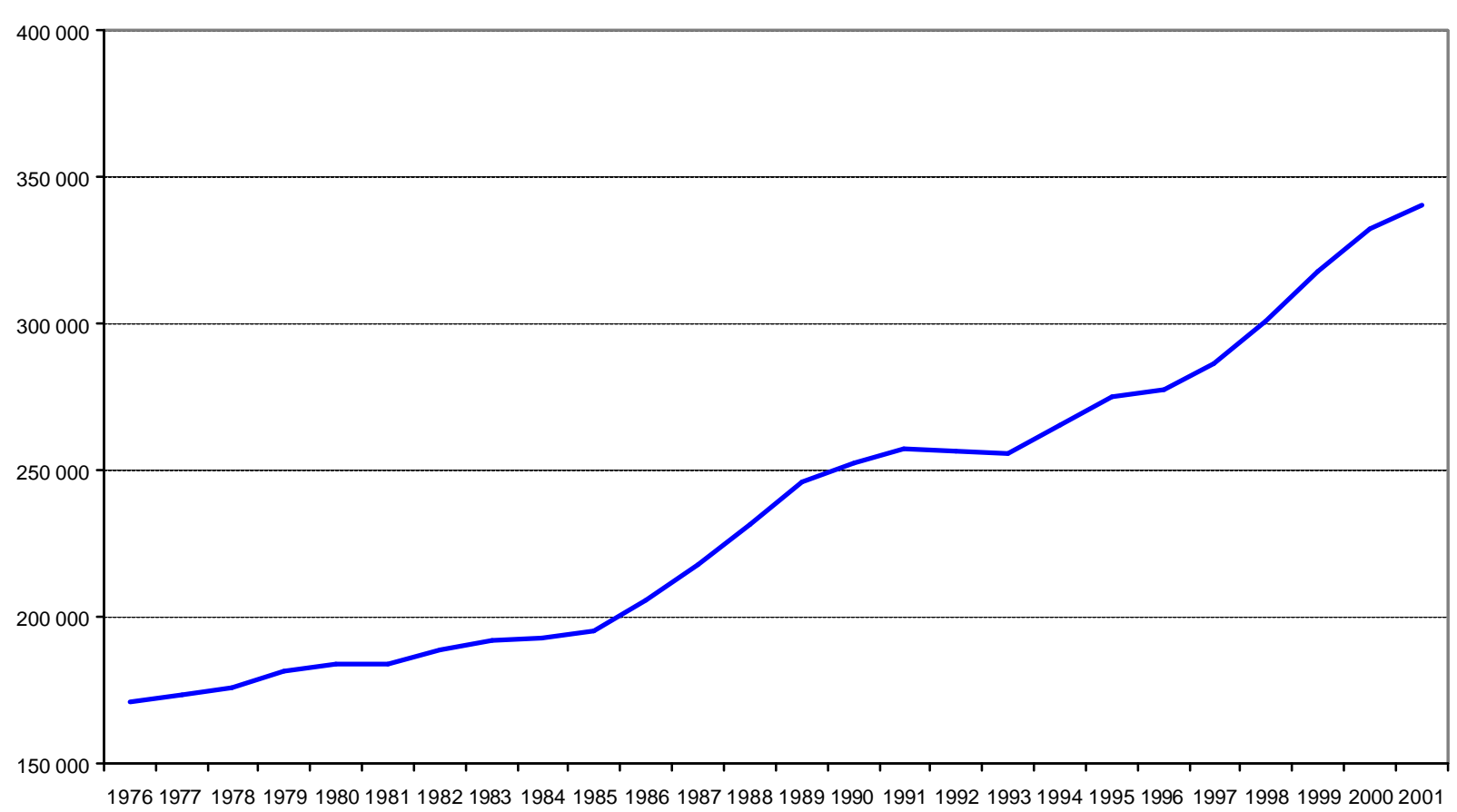

Figure 4: Evolution de l'emploi dans le transport routier de marchandise entre 1976 et 2001. Source : UNEDIC. 
Par ailleurs Emile Q uinet souligne dans son article que le nombre d'entreprises de plus de cent salariés est passé de 111 en 1986 à 170 en 1991, on assiste en effet depuis une dizaine d'années à un renforcement des opérateurs les plus grands. La croissance de l'emploi est continue dans les établissements de 10 salariés et plus et surtout dans ceux de 20 salariés et plus alors qu'il stagne dans les établissements les plus petits après la forte hausse survenant juste après 1986.

Malheureusement, ainsi que Q uinet le fait remarquer, cette déréglementation ne s'est pas faite sans heurts. Preuves en sont les grèves des routiers et les blocages des routes, expressions de crises sérieuses du secteur ${ }^{30}$. A l'époque, il s'était agi de déréglementer et de supprimer les entraves à l'exercice de la profession les plus patentes. Pourtant, le laisser-faire est souvent mauvais car il protège les plus puissants : dans le cas d'espèce, les grandes entreprises en place ont pu imposer leurs conditions aux petits transporteurs. En outre, le code de la route et le code du travail ont été souvent malmenés dans les plus petites entreprises, soumises à de fortes pressions concurrentielles. Cet exemple illustre le fait que la modification des règles de la concurrence ne doit pas se limiter à instituer le laissez-faire. Il faut mettre en œuvre des moyens pour que les règles limitant le pouvoir des grandes entreprises et protégeant les salariés soit respectées.

Les exemples du commerce et du transport routier indiquent clairement que certaines réglementations limitant la concurrence ont un impact négatif sur l'emploi et défavorisent le consommateur. Les études macroéconomiques suggèrent que de telles réglementations constituent une source importante du sous-emploi chronique en France.

L es conséquences macroéconomiques

Les études mobilisant des données internationales cherchent à établir un lien entre les entraves à la concurrence et le niveau de l'emploi observés dans chaque pays. La première tâche de ces travaux consiste à construire un indicateur unique résumant toutes les entraves de ce type, ce qui permet de classer les pays selon un indicateur de «degré de concurrence globale ». D eux études récentes, réalisées respectivement par l'O $\mathrm{CDE}^{31}$ et la Banque Mondiale, se sont livrées à cet exercic $e^{32}$. Dans l'étude de l'OCDE, la construction de cet indicateur tient compte, entre autres, du poids de l'Etat dans le capital des entreprises, de l'importance de la législation antitrust,

\footnotetext{
30 L'effet de la crise de 1995 se voit sur le graphique ci dessus. Il faut aussi se rappeler le mouvement de 1992.

${ }^{31}$ Paul Conway, Véronique Janod et Giuseppe Nicoletti (2005), "Product Market Regulation in OECD Countries: 1998 to 2003", OE CD E conomics D epartment W ork ing Papers, No. 419,

$32 \mathrm{D}$ oing business in 2006, World Bank, 2005.
} 
des pratiques de contrôle des prix, de la facilité à obtenir des licences d'exploitation, des barrières à l'entrée des firmes étrangères, de la structure des industries de réseaux et des contraintes administratives pour créer une entreprise. Selon cette étude, qui repose sur plusieurs milliers d'observations, ce sont les pays anglo-saxons qui présentent le moins d'obstacles à la concurrence. D ans la zone OCDE, les pays les plus «libéraux » sont, dans l'ordre décroissant, le Royaume-Uni, l'Australie, les Etats-Unis, la Nouvelle Zélande et le Canada. Mais les pays de l'Europe du nord les suivent d'assez près. En revanche, la France n'occupe que la 23ème place (sur 30), seules dans l'Union européenne la G rèce et l'Italie se trouvent derrière.

L'étude de la Banque Mondiale s'appuie sur un indicateur de «la facilité de la pratique des affaires » dont les critères sont sensiblement les mêmes que ceux de l'étude de l'OCDE. Il n'est donc pas surprenant que les classements des pays soient aussi sensiblement les mêmes. Ainsi, selon la Banque Mondiale les pays en tête pour la facilité du commerce sont, dans l'ordre et en ne retenant que les pays de la zone OCDE, la Nouvelle Zélande, les Etats-Unis, le Canada, la Norvège, l'Australie, le D anemark et le Royaume-Uni. La France occupe le $24^{\text {ème }}$ rang. Parmi les membres de l'Union européenne seule la Grèce est derrière. Ces deux études convergent vers le même constat: la France est un pays où les barrières réglementaires demeurent mportantes. Dans le graphique ci-dessous nous avons mis en relation, pour chaque pays et pour l'année 2004, l'indicateur de facilité du commerce construit par la Banque Mondiale et le taux d'emploi dans ce pays. En abscisse, l'indicateur «Rang» classe les pays par ordre croissant d'entraves réglementaires. En d'autres termes, le pays ayant le rang 1, en l'occurrence la Nouvelle Zélande est le pays où les entraves réglementaires sont les plus faibles. 


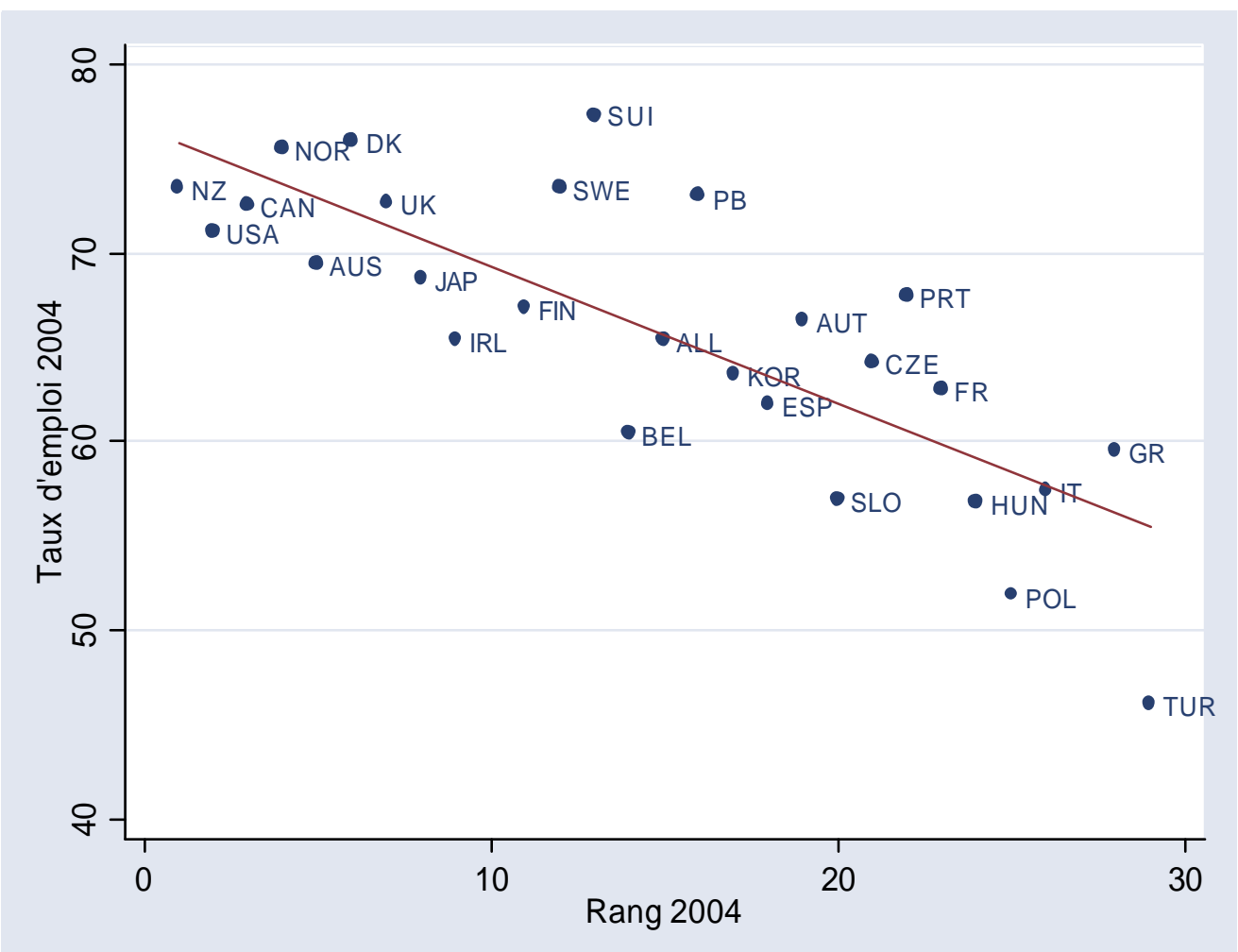

Figure 5 : Relation entre l'indicateur du degré de facilité du commerce et le taux d'emploi en 2004. Source : OCDE et Banque Mondiale.

Ce graphique montre que la corrélation entre le taux d'emploi et le Rang est très forte. Les pays qui ont le moins de barrières réglementaires sont aussi ceux où le taux d'emploi est le plus fort. Par exemple, les taux d'emplois des quatre premiers du classement, la Nouvelle Zélande, les Etats-Unis, le Canada et la Norvège, sont respectivement de 73,5\%, 71,2\%, 72,6\% et 75,6\%. En revanche, les taux d'emplois de la France, de la G rèce, de l'Italie et de la Turquie, qui se trouvent dans le bas du classement, sont de $62,8 \%, 59,6 \%, 57,4 \%$ et 46,1\%. Il est possible d'aller un peu plus loin que ces corrélations en estimant à l'aide d'un modèle statistique les effets d'une modification de l'indicateur de concurrence d'un pays sur les performances macroéconomiques de ce pays. Ainsi, l'étude de la Banque Mondiale montre, à l'aide d'une simulation numérique de ce type, que le taux de chômage d'un pays comme la Grèce pourrait être abaissé de plus de trois points de pourcentage si ce pays avait un cadre pour la vie économique équivalent à celui du D anemark. Une autre étude récente ${ }^{33}$ aboutit à un résultat sensiblement identique pour la France. Elle trouve que si la France alignait sa réglementation sur le pays de l'O CD E le plus «vertueux », elle gagnerait 1,2 points de pourcentage sur son taux d'emploi (ce qui correspond à 500000

${ }^{33}$ Nicoletti, G . et S. Scarpetta, «Regulation, productivity and growth: OECD evidence», E conomic Policy, avril 2003. 
emplois) et que le taux de croissance annuel de la productivité totale des facteurs augmenterait d'environ 0,2 point de pourcentage sur les dix prochaines années ${ }^{34}$.

Les conséquences négatives des barrières à l'entrée ne concernent pas que le niveau de l'emploi. Une étude récente ${ }^{35}$ portant sur 85 pays (les données couvrent principalement le nombre de procédures, les délais et les coûts que doit supporter un entrepreneur potentiel avant de pouvoir commencer légalement son activité) montre qu'un pays établissant plus de barrières est en général un pays où la corruption est plus forte, où la taille de l'économie informelle est plus grande, sans que pour autant la qualité des biens et des services, publics ou privés, soit améliorée.

Ainsi, corruption et entraves à la concurrence se confortent mutuellement. D es autorités publiques sous l'influence de puissants groupes de pression auront tendance à dresser plus de barrières à la concurrence. A l'inverse, les entraves à la concurrence produisent des « rentes »dont la pérennité nécessite parfois qu'une fraction en soit détournée au profit des représentants de l'autorité publique.

\section{Les principes d'une saine gestion de la concumence}

Une bonne gestion de la police et de la justice a pour but de protéger le plus grand nombre contre les agissements délictueux de quelques uns. Une régulation de la concurrence efficace doit ainsi viser à assurer des gains à l'ensemble de la société en limitant les possibilités de nuisances économiques d'une minorité. La régulation de la concurrence est, au même titre que la justice et la police, un bien public essentiel au bon fonctionnement d'une société moderne. En outre, la concurrence ne peut être réellement efficace que s'il existe d'autres institutions susceptibles d'assurer les perdants ${ }^{36}$. Une saine régulation de la concurrence doit donc reposer sur deux leviers : des autorités indépendantes jugeant des mesures à prendre pour que la concurrence ne soit pas faussée et un système assurantiel suffisamment développé.

D es autorités indépendantes

\footnotetext{
${ }^{34}$ Sur le plan théorique, les conséquences macroéconomiques de la suppression des entraves à la concurrence ont été analysées dans les articles de Blanchard, O. et Giavazzi, F., «Macroeconomic Effects of Regulations and Deregulation in G oods and Labour Markets », Q uarterly Journal of E conomics, 2003, 118(3), pp. 879-907; et Haefke, C. et Ebell, M., "The Missing Link: Product Market Regulation, Collective Bargaining and The European Unemployment Puzzle», mimeo, Universitat Pompeu Fabra, 2004.

${ }^{35}$ S. Djankov, R. La Porta, F. Lopez-de- Silanes et A. Shleifer, "The regulation of entry", Q uarterly Journal of E conomics, Février, 2002.

${ }^{36}$ En termes plus techniques, la concurrence n'est efficace que s'il existe un système complet de marchés.
} 
La régulation de la concurrence est une activité complexe pour au moins deux raisons. Tout d'abord, les infractions à la concurrence sont souvent difficiles à établir. L'histoire récente a par exemple montré qu'il est très difficile de prouver que Microsoft abuse d'une position dominante. D'autre part, l'intérêt général impose parfois de limiter certaines activités parce qu'elles exercent des effets négatifs sur l'ensemble de la société. Par exemple, le transport routier de marchandises est plus polluant et plus dangereux que le transport ferroviaire. L'Etat a ainsi quelques légitimités à taxer le premier secteur et à subventionner le second... ce qui constitue une distorsion à la concurrence. Ces difficultés rendent difficile l'application de principes simples, indiscutables, en matière de régulation de la concurrence $e^{37}$. Cet état de fait peut favoriser les pressions pour mettre en place des barrières réglementaires allant à l'encontre d'une concurrence harmonieuse, comme nous l'avons vu dans le cas du commerce de détail en France. Ainsi, l'un des enjeux majeurs de la régulation de la concurrence consiste à mettre en place des institutions qui protègent la société contre de telles pressions.

En France, le contrôle de la concurrence est assuré conjointement par le Conseil de la concurrence, qui est une institution indépendante de l'administration, et par la DGCCRF (Direction générale de la concurrence, de la consommation et de la répression des fraudes) qui dépend directement $\mathrm{du}$ ministère des finances. Ces deux institutions ont des fonctions différentes, mais ce dualisme est source de confusion et nuit à l'efficacité de la politique de la concurrence.

Organisme administratif créé en $1986^{38}$, le Conseil de la concurrence est une instance collégiale indépendante dont le président et les membres sont nommés par le ministre de

\footnotetext{
37 Voir sur ces thèmes : John K woka, et Lawrence White (éditeurs) The A ntitrust revolution, E conomics, Competition and Policy, Oxford University Press, quatrième édition, 2004.

${ }^{38}$ Créé par l'ordonnance du 1er décembre 1986, le Conseil de la concurrence a succédé à la Commission de la concurrence, créée en 1977, qui a elle-même succédé à la Commission technique des ententes et des positions dominantes. Le décret du 9 août 1953 a inséré dans l'ordonnance du 30 juin 1945 des dispositions sur les ententes illicites et a créé la Commission technique des ententes. Ces dispositions ont été étendues à l'abus de position dominante par la loi du 2 juillet 1963. La Commission technique des ententes et des positions dominantes avait pour mission de rendre des avis au ministre de l'économie sur les pratiques d'ententes et de positions dominantes. Ses avis pouvaient conduire le ministre à prononcer des sanctions pécuniaires ou à transmettre le dossier au juge pénal.
}

La loi du 19 juillet 1977 a créé la Commission de la concurrence qui s'est vue confier, par rapport à la Commission technique des ententes et des positions dominantes, deux attributions supplémentaires:

- conseiller le gouvernement sur toute question intéressant la concurrence - donner des avis sur les opérations ou projets de concentration

L'ordonnance de 1986 a introduit des novations importantes :

- le transfert du pouvoir de sanction du ministre au Conseil, assorti du contrôle du juge judiciaire

- l'élargissement, notamment aux entreprises, des possibilités de saisine

- une procédure garantissant mieux les droits des intéressés. 
l'économie. Selon l'ordonnance de 1986, qui a par ailleurs posé le principe général de la liberté des prix et de la concurrence, sa mission consiste à «instaurer une discipline de concurrence crédible pour les entreprises. En effet, les marchés ne peuvent concourir à l'efficacité économique que si des règles de droit garantissent aux opérateurs la liberté de fixer leurs prix, le libre accès au marché, mais aussi l'absence d'abus de puissance économique par ceux qui la détiennent ». Le Conseil de la concurrence disposent de réels pouvoirs pour réprimer les ententes, les abus de position dominante et les prix abusivement bas. Il peut prononcer des injonctions, prendre des mesures conservatoires, infliger des sanctions pécuniaires et accepter des engagements. Par ailleurs, il peut être amené à rendre des avis sur diverses questions de concurrence. D es parties privées peuvent déposer plainte contre d'autres entreprises directement auprès du Conseil de la concurrence et il peut être fait appel de ses décisions devant la Cour d'appel de Paris. Le Conseil de la concurrence compte 17 membres (siégeant essentiellement à temps partiel) et un secrétariat d'environ 120 personnes, dont 40 sont des rapporteurs permanents. Son budget annuel est de 8,5 millions d'euros. Le Conseil dispose donc de moyens très limités. Son homologue britannique, l'O ffice of fair trading, emploie 243 personnes et son budget s'élève à 47 millions d'euros.

Le Conseil de la concurrence n'a pas le pouvoir de mener des enquêtes. Elles sont l'apanage de la DGCRF. Cette dernière compte plusieurs milliers d'agents en poste dans l'ensemble du pays, qui s'occupent pour la plupart d'autres tâches relatives à la surveillance du marché et à la protection des consommateurs. La DGCCRF consacre moins de $10 \%$ de ses ressources aux questions de concurrence et de "pratiques restrictives ». Il faut noter que la DGRCF ouvre chaque année des milliers de petites procédures concernant les pratiques restrictives, contre tout au plus quelques centaines pour les pratiques anticoncurrentielles. Pour ce qui concerne le contrôle global des industries de réseaux, les compétences sont partagées entre le Conseil de la concurrence et la DGCRF. Mais pour tout le reste, le rôle du Conseil de la concurrence est simplement consultatif et, pour la plupart des opérations, son avis n'est pas demandé. En particulier, le contrôle des fusions, domaine sensible s'il en est, relève de la

Une loi du 11 décembre 1992 a habilité le Conseil de la concurrence à appliquer les articles 81 à 83 ( ex-85 à 87) du Traité de Rome. La loi du 1er juillet 1996 a élargi les attributions contentieuses du Conseil aux pratiques de prix abusivement bas. L'ordonnance du 18 septembre 2000 a inséré l'ordonnance du 1er décembre 1986 dans le livre IV du code de commerce.

Le 15 mai 2001, le volet concurrence de la loi sur les nouvelles régulations économiques a modifié le droit de la concurrence dans le but de :

- renforcer l'efficacité de la lutte contre les pratiques anticoncurrentielles

- garantir le respect du principe d'égalité des armes

- améliorer la coopération internationale

- contrôler les concentrations de manière plus systématique et plus transparente. 
compétence exclusive du ministère de l'économie. Le Conseil de la concurrence n'y a encore qu'un rôle consultatif qui devient cependant obligatoire en cas d'injonction et d'interdiction de fusion. Les décisions prises sont publiées et motivées dès lors qu'une atteinte à la concurrence a été détectée. Les décisions peuvent être déférées au Conseil d'État.

Cette architecture manque pour le moins de transparence. Ainsi, dans son rapport consacré à la France en 2005, l'OCDE note qu' «en ne soumettant pas au Conseil pour étude une opération potentiellement anticoncurrentielle, un ministre pourrait la soustraite à un examen attentif afin de répondre à d'autres objectifs politiques. ». De même, le fait que les enquêtes ne puissent être menées que par la DGCRF est un autre biais potentiel d'influence des pouvoirs publics. Une enquête gênante pour un ministère peut être tout simplement bâclées ou trâner en longueur pendant plusieurs années. Le système français de contrôle de la concurrence laisse donc trop la porte ouverte à des interventions discrétionnaires des autorités. Ainsi, rien ne garantit que les décisions en matière de fusion seront bien prises en fonction de l'intérêt général, d'autant que les grandes entreprises françaises sont souvent des entreprises publiques, ou des entreprises partiellement privatisées. Certains ministres se sont engagés à suivre les avis du Conseil, Dominique Strauss-Khan en fait partie, d'autres ont pris des décisions contraires aux avis du Conseil. Par exemple, Laurent Fabius a refusé l'achat de la branche cirage de Benckizer par Sara Lee alors que le Conseil y était favorable $e^{39}$. Le pouvoir du Conseil est également affaibli par un risque de sanction pénale à peu près nul, même pour les cas les plus graves d'atteinte au droit de la concurrence. En effet, selon le droit français, pour appliquer une sanction pénale il ne suffit pas de prouver que la personne incriminée a participé à une entente anticoncurrentielle, il faut prouver aussi qu'elle y a pris une part déterminante et frauduleuse. Selon Frédéric Jenny, qui fut viceprésident du Conseil de la concurrence, ces deux critères sont des obstacles quasi insurmontables et il conviendrait de les faire disparaitre de nos textes de loi $^{40}$. En pratique, les amendes sont ainsi les seules sanctions applicables. Mais des amendes, même très élevées (ce qui n'est d'ailleurs pas souvent le cas), peuvent toujours être plus ou moins payées ou différées. En revanche, une peine de prison fait beaucoup plus peur et peut s'avérer totalement dissuasive.

L'ensemble de ces arguments plaide pour une refonte simple mais profonde du système français de contrôle de la concurrence. La totalité des compétences en ce domaine devrait être transférée au Conseil de la concurrence avec les ressources que cela suppose dès lors, en particulier, que cet organisme serait chargé de mener ses propres enquêtes. De plus, le droit de la

\footnotetext{
${ }^{39}$ Ces informations sont extraites d'un article de Nathalie Brafman paru dans le journal L e M onde du 20 septembre 2005 (page 18).

40 Interview au journal L e M onde du 20 septembre 2005.
} 
concurrence devrait aussi être réformé afin de permettre une application effective des sanctions pénales pour des délits constituant des entraves à la concurrence.

\section{En guise de conclusion : concilier sécurité et concurrence}

Un monde incertain

Les innovations font naitre de nouveaux produits, de nouvelles firmes, de nouvelles industries et font disparaitre les procédés anciens. Une économie de marchés où règne la concurrence est perpétuellement agitée par les tentatives des entreprises de s'approprier de nouveaux débouchés. Certaines de ces tentatives connaissent le succès, en général temporaire, la concurrence ne permet aucun repos, d'autres échouent. La concurrence engendre un bouillonnement d'essais et d'erreurs, de succès et d'échecs, de créations et de destructions. Par essence, ce bouillonnement est imprévisible, et c'est pourquoi il n'existe pas (en tous les cas pas à la même échelle) dans une économie planifiée. Une économie de marché se caractérise par une incertitude permanente et, surtout, inévitable, car consubstantielle à la concurrence que se livrent les entrepreneurs. Très certainement, la succession des innovations augmentera sans relâche la richesse collective, mais nous ne savons pas quels seront ses effets sur l'avenir de chacun d'entre nous. Une société où les robots réaliseront des tâches de plus en plus complexes sera plus riche que celles qui l'ont précédée, mais aura-t-elle besoin de plus ou de moins de travailleurs peu qualifiés ? Nul ne le sait. Le développement des logiciels de traduction automatique fera-t-il disparaitre les interprètes et les traducteurs ? Nul ne le sait. Et même si ces professions devaient effectivement disparaitre nul ne sait à quel horizon cela se produira.

C'est le caractère imprévisible de l'économie de marché qui la rend insupportable à beaucoup, pour ne pas dire au plus grand nombre. Il est en effet difficile de percevoir que la croissance des richesses est indissociable de l'incertitude radicale qu'engendre la concurrence. Cette incertitude radicale est d'autant plus insupportable qu'elle peut entrainer, à terme, une répartition très inégale des richesses ${ }^{41}$. Le mouvement permanent de créations et de destructions produit des gains pour la collectivité toute entière, mais ils ne sont pas forcément distribués selon les mérites et les responsabilités de chacun. L'ouvrière d'une usine du textile traditionnel qui perd son emploi du fait de la concurrence des pays à bas salaires n'en est pas responsable. Le chef de rayon de Marks et Spencer, qui voit disparaitre le magasin dans lequel il travaille, n'est pas

${ }^{41}$ Une économie de marché non régulée peut connaitre bien d'autres échecs, en particulier pour tout ce qui touche à
l'environnement et au bien être des générations futures. Le livre de Roger Guesnerie, L'E conomie de marché, 
responsable du changement des goûts des consommateurs. Le processus de destruction créatrice produit inévitablement ce type d'aléa. Aujourd'hui, des millions de personnes bénéficient peu de la croissance économique, même dans les pays les plus riches. A elle seule, la France compte plus d'un million d'allocataires du RMI dont une très grande majorité est exclue de fait du marché du travail. Intrinsèquement, le marché reste une formidable machine à créer des richesses, mais aussi à exclure. Ces deux phénomènes sont indissociables; en suscitant la recherche du profit, le marché enrichit certains, mais appauvrit d'autres, souvent ceux qui sont déjà les plus pauvres au départ.

Il est vraisemblable que la répartition des risques soit devenue plus inégalitaire au cours des vingt dernières années. Au sein des travailleurs, mais aussi entre les actionnaires et l'ensemble des salariés Une étude récente de David Thesmar et Mathias Thoening montre que, paradoxalement, en France, à partir de la fin des années 1980, le risque d'entreprise serait de moins en moins pris en charge par les actionnaires... et de plus en plus par les salariés ${ }^{42}$ ! Fondamentalement, ce phénomène serait dû à la moindre importance des entreprises familiales et à la montée en puissance des entreprises appartenant à des actionnaires multiples, contrôlées, en fait, par des fonds d'investissement. Ces derniers sont beaucoup plus à même de diversifier les risques (ce qui est bon pour l'actionnaire), mais accroît fortement la volatilité des profits et des emplois pour chaque entreprise (ce qui est moins bon pour le salarié de l'entreprise en question). L'étude de David Thesmar et Mathias Thoening montre que cette volatilité se transmet à l'ensemble des firmes, qu'elles soient cotées en bourse ou non. Le résultat est que les travailleurs pris dans leur ensemble, subissent plus le risque d'entreprise que l'ensemble des actionnaires. Pour «compenser» cette nouvelle fonction des travailleurs de ce nouveau rôle, on pourrait songer à augmenter les salaires. Mais Philippe Martin, professeur d'économie à l'université de Paris 1, note à juste titre que ce transfert du risque ne s'est pas accompagné d'une modification dans le partage de la valeur ajoutée. D epuis 10 ans, le «travail »s'attribue environ deux tiers des richesses produites et le «capital » récolte le tiers restant. Toute hausse des salaires modifierait vraisemblablement le partage de la valeur ajoutée à court terme, mais les entreprises réagiraient en diminuant progressivement leur niveau d'emploi et le partage de la valeur ajoutée ne serait ainsi pas modifié sur le long terme. Le conflit entre le capital et le travail n'est donc pas aujourd'hui celui d'un partage de la valeur ajoutée mais celui d'un partage du risque ${ }^{43}$.

Flammarion, collection D ominos, 1996, dresse un bilan exhaustif des succès et des échecs de ce mode d'organisation des échanges. Roger Guesnerie est professeur au Collège de France.

42 «Financial Market D evelopment and the Rise in Firm Level Uncertainty », Mimeo, octobre 2004.

43 Philippe Martin, "Capital et travail, inégalité des risques », article paru dans le journal L ibération, le 4 juillet 2005, p. 37. 
D e façon générale, les conflits liés au partage du risque, que ce soit entre les travailleurs et les détenteurs du capital, ou entre les travailleurs eux-mêmes, ne peuvent trouver de solutions qu'à travers un développement important des institutions permettant d'assurer l'ensemble des échanges, surtout sur le très long terme. Dans une société où la richesse collective augmente en permanence, il est possible d'assurer convenablement chaque personne en diluant le plus possible les risques au sein de l'ensemble du corps social. Encore faut-il imaginer les institutions qui permettent effectivement ce partage efficace du risque ${ }^{44}$. En l'absence de telles institutions, les conflits autour du partage du risque ne feront qu'accentuer les comportements de repli sur les «rentes » existantes. A l'inverse, l'avènement d'institutions susceptibles de mieux assurer les individus tout au long de leur vie rend moins profitables les luttes pour la préservation de ces rentes de situation et permet donc de mettre en œuvre plus facilement les réformes nécessaires. Dans le domaine de l'emploi, la réflexion sur ce point a beaucoup progressé au cours de ces dernières années. A l'idée de sécurisation des emplois, incompatible avec le processus de destruction créatrice que nous avons abondamment évoqué jusque-là, elle tend aujourd'hui à lui préférer celle de «sécurisation des parcours professionnels» ou de «Sécurité Sociale Professionnelle.

La « Sécurité Sociale » instituée en France par les ordonnances de 1945 avait pour objectif de garantir «à chacun qu'en toutes circonstances il disposera des moyens nécessaires pour assurer sa subsistance et celle de sa famille dans des conditions décentes. Trouvant sa justification dans un souci élémentaire de justice sociale, elle répond à la préoccupation de débarrasser les travailleurs de l'incertitude du lendemain, de cette incertitude constante qui crée chez eux un sentiment d'infériorité et qui est la base réelle et profonde de la distinction des classes entre les possédants sûrs d'eux-mêmes et de leur avenir et les travailleurs sur qui pèse, à tout moment, la menace de la misère ${ }^{45}{ }$. Certes, une Sécurité Sociale Professionnelle aussi performante soit elle ne pourra garantir un emploi pour chacun à chaque instant de sa carrière, tout comme la «Sécurité Sociale » ne peut garantir une guérison instantanée pour tous dans le domaine de la santé. La «Sécurité Sociale » doit garantir l'accès à des soins de qualité pour tous. D ans le domaine de l'emploi, la «Sécurité Sociale» devrait garantir un revenu décent et un accompagnement de qualité de tous les demandeurs d'emploi en permettant une reconversion vers les métiers d'avenir.

44 Pour l'ensemble des activités économiques, Robert Shiller a esquissé ce que pourrait être ces institutions susceptibles de fournir une «assurance toute au long de la vie». Voir The N ew Financial Order, Princeton University Press, 2003.

${ }^{45}$ Extrait de l'exposé des motifs de l'ordonnance du 4 octobre 1945. 
Cette «sécurisation» suppose une réforme coordonnée visant deux objectifs: améliorer le reclassement en affirmant le rôle de l'Etat et supprimer les statuts d'emploi précaires en créant un contrat de travail unique à durée indéterminée ${ }^{46}$.

Dans un tel système, l'Etat devrait avoir pour rôle de coordonner l'ensemble des processus de reclassement, ce qui suppose qu'il s'en donne les moyens. Précisément, pour mieux accompagner les chercheurs d'emplois, le rapprochement des diverses institutions chargées du traitement du chômage s'impose. En particulier, la dualité de structure entre ANPE et UNEDIC doit être supprimée afin de mettre en place un guichet unique indispensable à un suivi cohérent des demandeurs d'emploi. Il faut aussi poser le principe d'un traitement différencié des chômeurs. Toute personne entrée au chômage doit être évaluée de façon personnalisée et les dépenses doivent être concentrées sur les personnes qui en ont le plus besoin et seulement sur elles. Ce service public de placement rénové et renforcé doit se substituer à l'obligation de reclassement des entreprises. En contrepartie, les entreprises participent au financement du service public de l'emploi par le biais d'une taxe sur licenciements. La mutualisation des ressources autour du service public constitue une garantie de solidarité. Elle permet aussi de poursuivre la professionnalisation des activités de placement et de reclassement; les opérateurs étant rémunérés en fonction de spécificités du chômeur et de la réussite de la ré-insertion dans l'emploi.

La sécurisation des parcours professionnels nécessite aussi une profonde réforme de la formation professionnelle. La recherche d'une plus grande efficacité suppose qu'elle soit intégrée au service public de l'emploi et que les organismes qui dispensent de la formation professionnelle soient rémunérés aux résultats après avoir été mandatés par le service public. La mobilité de la main-d'œuvre peut être grandement facilitée par la certification des compétences professionnelles des salariés. Le principe de validation des acquis de l'expérience (VAE) introduite par la loi de modernisation sociale en janvier 2002 est une étape importante dans cette direction. La VAE permet de certifier des connaissances acquises dans le cadre d'une activité professionnelle. Elle a cependant encore des défauts (nombreux dossiers retirés, peu de dossiers acceptés, trop d'exigence dans les matières générales) qui peuvent être supprimés en mettant en place des validations automatiques des acquis, afin d'obtenir en particulier le CAP, à partir d'une expérience minimum (trois ans, par exemple, comme pour les plombiers).

Enfin, la création d'un contrat de travail unique à durée indéterminée, et donc la suppression de tous les statuts d'emplois précaires, permettrait une meilleure mutualisation des risques. Elle suppose néanmoins une réforme importante du droit du travail. La césure CDD-

\footnotetext{
${ }^{46}$ Ces thèmes sont développés dans le rapport de Pierre Cahuc et Francis Kramarz : D e la précarité à la mobilité: vers une
} 
$\mathrm{CD}$ I et la réglementation des licenciements économiques entrainent de profondes inégalités : les jeunes sont cantonnés à des emplois en $\mathrm{CD}$ D, et les entreprises hésitent à embaucher des seniors sur des emplois stables, car leur destruction est très coûteuse. Le licenciement économique est accompagné de procédures de reclassement formellement exigeantes mais souvent contournées au détriment des salariés les plus fragiles et les moins informés. Ce système aboutit à un partage très inégalitaire des risques de perte d'emploi. Il pousse aussi à conserver trop longtemps des emplois peu productifs et à détruire trop d'emplois efficaces, notamment du fait de l'interdiction de renouvellement des CDD. La suppression du CDD et la création d'un contrat de travail unique permettent de lever ces difficultés. Un tel contrat devrait avoir trois composantes : il doit être à durée indéterminée ; il doit donner droit à une prime fonction de l'ancienneté en cas de licenciement (le montant payé inclurait en particulier la composante «précarité » versée en fin de $\mathrm{CDD}$ ) ; il doit donner lieu à une taxe sur les licenciements qui servira à garantir le droit au reclassement du salarié, reclassement assuré non plus par les entreprises mais par le service public de l'emploi. 